\title{
Vitalidade Fetal em Gestaçoes Complicadas com Diabete Melito Pré-Gestacional: Um Estudo Longitudinal
}

\author{
Fetal Surveillance In Pregnancies With Pregestational Diabetes \\ Mellitus: A Longitudinal Study
}

Roseli Mieko Yamamoto Nomura, Rossana Pulcineli Vieira Francisco, Carlos Alberto Maganha, Seizo Miyadahira, Jorge D. Banduki Neto, Marcelo Zugaib

\begin{abstract}
RESUMO
Objetivos: estabelecer parâmetros dos testes de vitalidade fetal em gestações com diabete melito pré-gestacional que se correlacionam com a ocorrência de recém-nascidos (RN) grandes para idade gestacional (GIG).

Métodos: entre março de 1999 e junho de 2001, 46 gestações simples sem malformações com diabete melito pré-gestacional foram acompanhadas prospectivamente e foram realizados semanalmente, a partir da $28^{a}$ semana: perfil biofisico fetal (PBF), indice de liquido amniótico (ILA) e dopplervelocimetria das artérias umbilical e cerebral média. Os RN foram classificados como GIG quando apresentavam peso superior ao percentil 90. Testes estatísticos: teste exato de Fisher e t de Student.

Resultados: a média da idade gestacional no nascimento foi de 37,6 semanas e 15 RN foram GIG $(32,6 \%)$. Os resultados do ILA demonstraram média significativamente superior nos casos com RN GIG, nas semanas: $32^{a}(16,5 \mathrm{~cm}, p=0,02), 33^{a}(16,7 \mathrm{~cm}, p=0,03), 34^{a}(17,0 \mathrm{~cm}$, $p=0,02), 35^{a}(17,9 \mathrm{~cm}, p=0,000), 36^{a}(15,8 \mathrm{~cm}, p=0,03)$ e $37^{a}(17,5 \mathrm{~cm}, p=0,003)$. Nos RN não GIG, os resultados do ILA demonstraram as seguintes médias: $13,5 \mathrm{~cm}$ (32 ${ }^{a}$ semanas), 13,1

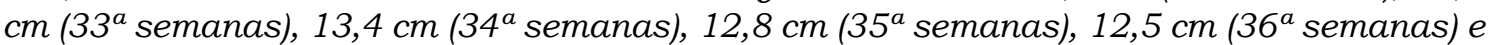
$12,8 \mathrm{~cm}$ ( $37^{a}$ semanas). Constatou-se associação significativa entre a ocorrência de valores superiores ou iguais a 18,0 cm no ILA e RN GIG, nas semanas: $34^{a}$ (60\%, $\left.p=0,03\right), 35^{a}$ (71,4\%, $p=0,01), 36^{a}(80 \%, p=0,02)$ e $37^{a}(66,7 \%, p=0,04)$. Nos $R N$ não GIG, verificou-se que as proporções de casos com ILA de valor superior ou igual a 18,0 cm, por semana, foram:

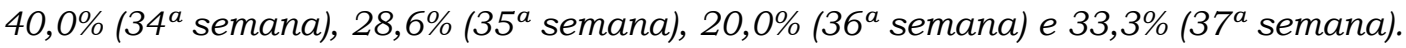

Conclusões: as alterações do ILA, principalmente aqueles superiores a $18,0 \mathrm{~cm}$, estão relacionadas ao RN GIG. A terapêutica materna deve ser ajustada para que se obtenha o melhor resultado possivel para o binômio mãe-feto, de acordo com os valores do ILA calculado durante a gestação.
\end{abstract}

PALAVRAS-CHAVE: Diabete melito. Índice de líquido amniótico. Perfil biofísico fetal. Dopplervelocimetria. Artérias umbilicais. Artéria cerebral média.

Setor de Vitalidade Fetal - Clinica Obstétrica - Hospital das Clinicas da Faculdade de Medicina da Universidade de São Paulo

Correspondência:

Roseli Mieko Yamamoto Nomura

Rua General Canavarro, 280 - Bairro Campestre

09070-440 - Santo André - SP

Tel:4991-2481 (res) 4221-8778 (com)

Fax: 4991-7312 (res) 4221-8752 (com)

e-mail: roselinomura@uol.com.br, vitalidade@hcnet.usp.br

Home Page: http://www.hcnet.usp.br/ob/

\section{Introdução}

A avaliação da vitalidade fetal no acompanhamento das gestantes diabéticas, principalmente das que apresentam este diagnóstico anteriormente à gestação, tem como objetivo principal assegurar o bem estar do feto. Atualmente porém, observa-se que outros aspectos podem ser 
avaliados dentro da rotina da propedêutica fetal, pois o controle glicêmico materno durante a gestação pode refletir-se em determinados parâmetros analisados por estes exames.

Atualmente, o rigoroso controle materno baseado no ajuste da insulinoterapia de acordo com o perfil glicêmico da paciente permite a continuidade da gestação até o termo, com seguran$\mathrm{ça}^{1-3}$. A partir de protocolos desenvolvidos na década de 70 , foram adotadas estratégias de controle materno e fetal, inclusive com hospitalização da paciente no final da gestação. Observou-se redução na mortalidade perinatal para valores inferiores a $5 \%$, e atualmente a taxa de perdas fetais apresenta índices inferiores a 2,5 por 1000 gestações ${ }^{4,5}$.

A fisiopatologia do sofrimento fetal na gestação com diabete melito está relacionada, principalmente, à resposta metabólica fetal frente à oferta elevada de glicose pela circulação materna. A hiperglicemia fetal promove o estado de hiperinsulinismo, com aceleração do crescimento e aumento do depósito de glicogênio e de lipides ${ }^{6}$. O aumento do metabolismo celular tem como conseqüência maior consumo de oxigênio nos tecidos, e, ao mesmo tempo, a glicosilação da hemoglobina fetal desloca a sua curva de dissociação para a esquerda, com aumento da afinidade pelo oxigênio ${ }^{7}$. Nesta situação temos, nos tecidos fetais, maior demanda de oxigênio e maior dificuldade na liberação do mesmo, facilitando a ocorrência da hipoxia tecidual. Estudos experimentais demonstram que a hipoxemia fetal leve pode resultar em acidose lática e óbito do feto, quando associada ao estado de hiperglicemia ${ }^{8}$. Portanto, o controle glicêmico materno durante a gravidez favorece a manutenção do estado metabólico fetal em condições adequadas para preservar sua vitalidade.

Outra anormalidade desencadeada pelo hiperinsulinismo fetal consiste na eritropoese extramedular, em resposta às alterações nos padrões de crescimento do feto $^{3}$, levando à poliglobulia e aumento da viscosidade sangüinea. Estas alterações podem influenciar os padrões de velocidade de fluxo na circulação fetal, fato este possivel de ser avaliado pela dopplervelocimetria9. Tornase interessante a possibilidade de identificar os fetos de maior risco para apresentar hiperinsulinemia e, portanto, desenvolver ganho ponderal excessivo e macrossomia no termo. Estes terão como conseqüência maior risco de complicações no parto e morbidade neonatal elevada.

O estado hiperglicêmico materno acompanha-se também de distúrbios na produção e reabsorção de líquido amniótico. A diurese osmótica fetal é descrita como um dos mecanismos impli- cados no desenvolvimento do polidrâmnio em gestações complicadas pelo diabete melito ${ }^{10}$. A avaliação do volume de líquido amniótico consiste em propedêutica rotineira na avaliação fetal, geralmente acessivel para a maioria das gestantes de alto risco, principalmente aquelas portadoras de diabete.

Outros aspectos importantes devem ser considerados no acompanhamento das gestações com diabete melito pré-gestacional. Pacientes com diagnóstico há longa data ou com vasculopatias, doenças renais ou hipertensão podem apresentar insuficiência placentária, com diversos graus de comprometimento da placenta ${ }^{11}$. Nestes casos, a dopplervelocimetria da circulação útero-placentária e da circulação fetal permite o diagnóstico de restrição do crescimento fetal e/ou sofrimento fetal, que pode agravar-se quando acompanhado pelas alterações metabólicas do diabete melito.

A avaliação da vitalidade fetal torna-se, portanto, um desafio, em face da complexidade dos aspectos maternos e fetais envolvidos. Este estudo foi delineado para se aprimorarem os métodos propedêuticos atualmente disponiveis para avaliação de fetos de gestantes com diabete melito pré-gestacional ${ }^{12}$. Assim, o objetivo deste trabalho consiste em estabelecer parâmetros que possam identificar, a partir do seguimento prospectivo longitudinal semanal destas gestantes, o periodo e as alterações dos testes de vitalidade fetal que se correlacionam com a ocorrência de recém-nascidos grandes para a idade gestacional, pois estes são os que apresentam maior risco de complicações metabólicas tanto no período antenatal como no pós-natal.

\section{Pacientes e Métodos}

Cinqüenta e uma gestantes com diagnóstico de diabete melito pré-gestacional foram acompanhadas prospectivamente, em estudo longitudinal, pelo Setor de Vitalidade Fetal da Clínica Obstétrica do Hospital das Clinicas da Faculdade de Medicina da Universidade de São Paulo (HCFMUSP), no período de março de 1999 a junho de 2001. Este estudo foi aprovado pela Comissão de Ética para Análise de Projetos de Pesquisa (CAPPesq) do HC-FMUSP.

Os critérios de inclusão foram: diagnóstico de diabete melito realizado anteriormente à gestação atual, gestações simples, acompanhamento pré-natal neste hospital e consentimento em participar do presente estudo. Foram excluídos da presente análise três casos nos quais se diagnosticou má formação fetal e dois cujos partos não 
foram realizados nesta instituição, totalizando casuística de 46 pacientes.

Vinte e cinco pacientes apresentavam diagnóstico pré-gestacional de diabete melito do tipo I (insulino-dependente) e 21 do tipo II (não insulinodependente). Em relação à cor da gestante, 34 $(73,9 \%)$ eram brancas, 11 (23,9\%) pardas e uma $(2,2 \%)$ negra. A idade materna variou de 16 a 45 anos, com média de 30,1 e desvio padrão de 7,5 anos. Em relação aos antecedentes obstétricos, 13 eram primigestas (28,3\%), $10(21,7 \%)$ secundigestas e $23(50,0 \%)$ apresentavam três ou mais gestações. Quanto às doenças associadas, quatro gestantes com diabete melito do tipo II e três com do tipo I apresentavam concomitantemente o diagnóstico de hipertensão arterial $(15,2 \%)$ e três pacientes $(6,5 \%)$ com diabetes do tipo I apresentavam também hipotiroidismo.

O acompanhamento pré-natal foi realizado pelo Setor de Endocrinopatias e Gestação, e o controle glicêmico baseou-se na mensuração dos valores de glicemia capilar total de jejum (normal quando entre 70 e $90 \mathrm{mg} / \mathrm{dL}$ ) e pós-prandial de duas horas (normal entre 70 e $120 \mathrm{mg} / \mathrm{dL}$ ). As medidas adotadas para obtenção do controle glicêmico incluíram a dieta fracionada de 35 a 45 $\mathrm{cal} / \mathrm{kg}$ de peso ideal por dia e insulinoterapia quando necessária. Iniciou-se com dose única matinal de insulina NPH, que foi ajustada progressivamente no decurso da gestação, acrescentando-se ou não doses noturnas e/ou insulina de ação rápida pré-prandial. As pacientes cujo controle se revelasse dificil foram hospitalizadas até acerto da dose de insulina.

A partir da $28^{a}$ semana de gestação, as pacientes foram encaminhadas ao Setor de Vitalidade para iniciarem a propedêtica fetal semanal. A partir do momento da hospitalização da paciente, as pacientes foram avaliadas com menor periodicidade: no minimo três vezes por semana. Os seguintes exames foram realizados: perfil biofísico fetal (PBF), índice do líquido amniótico, dopplervelocimetria da artéria umbilical e dopplervelocimetria da artéria cerebral média (ACM).

O PBF baseou-se na análise dos seguintes parâmetros: cardiotocografia, movimentos respiratórios fetais, movimentos corpóreos fetais, tônus fetal e índice de líquido amniótico (ILA). Durante o período máximo padronizado para observação das atividades biofísicas (30 minutos), foi caracterizada como normal a ocorrência de no mínimo um episódio de movimento respiratório com duração minima de 30 segundos. Os movimentos corpóreos fetais foram classificados como normais quando o feto apresentou, no mínimo, um movimento amplo ou três movimentos lentos, e o tônus, como normal, na presença de movimentos corpóreos fetais ou de acordo com os movimentos de abertura e fechamento das mãos durante o período de observação. O volume de líquido amniótico foi caracterizado como normal no $\mathrm{PBF}$ quando o ILA apresentou valor superior a $5,0 \mathrm{~cm}$. Cada parâmetro do PBF recebeu o escore 2 quando normal e o escore 0 quando anormal, sendo classificado de acordo com o seu somatório em: normal (8 ou 10), suspeito (6) ou alterado (4, 2 ou 0$)$.

O ILA foi avaliado sempre pelas mesmas pesquisadoras (RMY Nomura e RPV Francisco), de acordo com o método proposto por Phelan et $a{ }^{13}$. $\mathrm{O}$ volume de LA foi ainda caracterizado como aumentado quando apresentou ILA com valor superior ou igual a $18,0 \mathrm{~cm}^{14}$. Quando mais de uma medida foi realizada na mesma semana da gestação, foi calculada a média dos valores.

A dopplervelocimetria foi realizada por via transabdominal, utilizado-se equipamento de ultrasonografia da marca Toshiba SSA350A, com dispositivo de Doppler e mapeamento de fluxo a cores. Os exames foram realizados com a paciente em posição semi-sentada e os sonogramas dos vasos analisados foram obtidos com as imagens congeladas durante a inatividade fetal e em periodos de apnéia. Foram utilizados filtros de baixa freqüência (50 hertz) e analisados sonogramas com ondas uniformes. Para a obtenção do sonograma das artérias umbilicais, foi analisado o cordão na porção próxima à inserção placentária, sendo calculado o índice de pulsatilidade.

A avaliação dopplervelocimétrica da ACM foi realizada em corte transverso do pólo cefálico fetal, na altura dos tálamos, com o transdutor deslocado obliquamente, em direção à base do crânio. Neste ponto a artéria é facilmente identificada como um ramo maior do polígono de Willis que se dirige ântero-lateralmente em direção à fissura de Sylvius. Evitou-se a compressão do abdome materno, uma vez que a compressão do pólo cefálico fetal pode levar a alterações nos exames realizados nas artérias intracranianas. O cursor foi colocado na sua porção proximal, procurando-se alinhar o cursor na mesma angulação da ACM, de tal modo que o ângulo fosse o mínimo possivel, e sempre inferior a 20 graus. Foi calculado o índice de pulsatilidade e a velocidade máxima na sístole, com correção do ângulo quando necessário ${ }^{15}$.

As avaliações semanais dos testes de vitalidade foram comparadas com a adequação do peso do recém-nascido (RN). Para tanto, os RN foram classificados como pequeno para a idade gestacional (PIG), quando apresentasse peso inferior ao percentil 10 para a idade gestacional; como adequado para idade gestacional (AIG), se estivesse entre os percentis 10 e 90 , e como grande para a idade gestacional (GIG), quando com peso supe- 
rior ao do percentil 90, de acordo com a curva de normalidade de $\operatorname{Ramos}^{16}$, desenvolvida pelo setor de neonatologia deste hospital.

Foram também estudados os seguintes resultados neonatais: idade gestacional no nascimento, índices de Apgar de $1^{\circ}$ e $5^{\circ}$ minuto, peso do $\mathrm{RN}$, tipo de parto e internação em unidade de terapia intensiva neonatal. A idade gestacional foi calculada a partir da data da última menstruação quando esta foi compativel com exame ultrasonográfico realizado até a $20^{\mathrm{a}}$ semana. Quando a paciente não sabia referir a data da última menstruação ou se houvesse discordância com o exame ultra-sonográfico, a datação da gestação era baseada na primeira ultra-sonografia realizada.

Para as variáveis qualitativas utilizou-se o teste exato de Fisher. Para as variáveis quantitativas, utilizou-se o teste $t$ de Student para comparação das médias. Adotou-se como nivel de significância o valor $0,05(\alpha=5 \%)$. Com isso, niveis descritivos ( $\mathrm{p}$ ) inferiores a esse valor foram considerados significantes $(\mathrm{p}<0,05)$. Para cada semana de gestação analisada, os casos que não foram submetidas ao exame neste periodo foram excluídos da análise.

\section{Resultados}

$\mathrm{Na}$ população analisada, não ocorreu nenhum caso de óbito fetal ou neonatal. Vinte e seis RN $(56,5 \%)$ foram classificados como AIG, 15 $(32,6 \%)$ como GIG e 5 (10,9\%) como PIG. As médias do peso ao nascimento, nestes grupos de RN, foram, respectivamente, $2.885 \mathrm{~g}, 3.905 \mathrm{~g} \mathrm{e}$ $2.144 \mathrm{~g}$, com desvios padrões correspondentes de $537,3 \mathrm{~g}, 490,1$ g e 218,8 g.

A média da idade gestacional no nascimento foi de 37,6 semanas, com desvio padrão de 2,1 semanas. Não se observou diferença estatisticamente significativa entre as médias da idade gestacional de acordo com a adequação do peso dos RN. Os classificados como AIG apresentaram média de 37,5 semanas, com desvio padrão de 2,4 semanas; os GIG, média de 38,1 semanas, com desvio padrão de 1,7 semanas, e os PIG, média de 37,4 semanas, com desvio padrão de 2,2 semanas. $\mathrm{Na}$ maioria dos casos o parto ocorreu no termo (Figura 1) e o tipo de parto mais freqüente foi a cesárea, em 35 casos $(76,0 \%)$.

A distribuição dos resultados do PBF realizado a cada semana de gestação pode ser observada na Figura 2. Constata-se que, em quase todas as ocasiões, o resultado deste exame foi normal, isto é, PBF com valor 8 ou 10. Em apenas duas situações, caracterizou-se o PBF como 6 (suspeito), sendo indicada a resolução da gravidez.

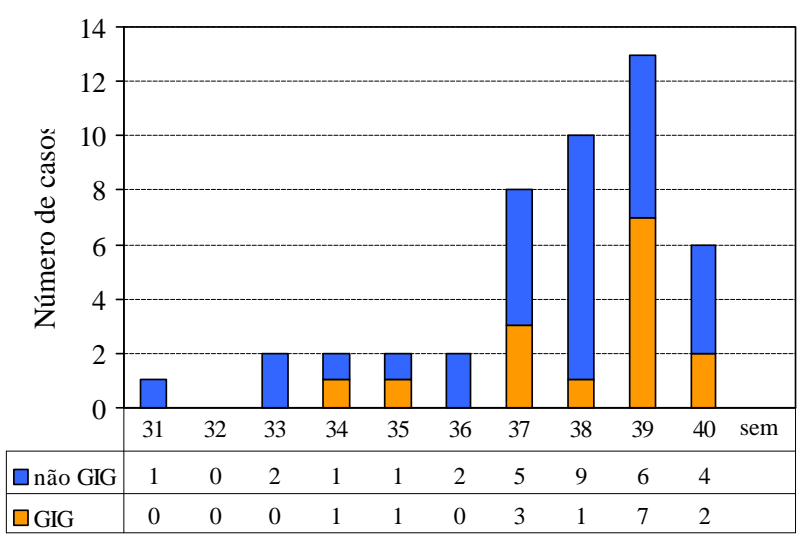

Figura 1 - Distribuição dos casos de acordo com a idade gestacional no nascimento, nos grupos GIG (grande para idade gestacional) e não GIG, em gestações com diabete melito pré-gestacional.

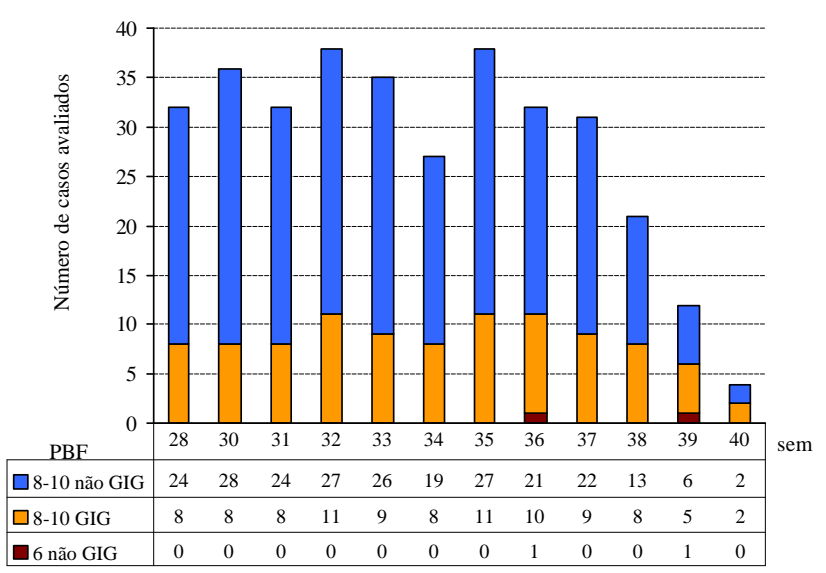

Figura 2 - Distribuição dos casos segundo o resultado do perfil biofísico fetal (PBF) nas idades gestacionais, nos grupos GIG (grande para idade gestacional) e não GIG, em gestações com diabete melito pré-gestacional.

Os resultados do ILA estão expostos na Tabela 1 . Observa-se que, avaliando os dados em cada semana estudada, a média do ILA foi significativamente superior nos casos com RN GIG, nas seguintes idades gestacionais: $32^{\mathrm{a}}, 33^{\mathrm{a}}, 34^{\mathrm{a}}, 35^{\mathrm{a}}, 36^{\mathrm{a}}$ e $37^{\mathrm{a}}$ semana, quando comparados aos casos não GIG, nas mesmas semanas de gestação. Classificando-se o valor do ILA, constata-se associação estatisticamente significativa entre a ocorrência de valores superiores ou iguais a $18,0 \mathrm{~cm}$ e o nascimento de RN GIG, nas seguintes idades gestacionais: $34^{\mathrm{a}}, 35^{\mathrm{a}}, 36^{\mathrm{a}}$ e $37^{\mathrm{a}}$ semana.

$\mathrm{Na}$ Tabela 2 pode-se observar os principais resultados da dopplervelocimetria das artérias umbilicais e cerebral média. Não foram constatadas diferenças significativas entre as médias do indice de pulsatilidade das artérias umbilicais e cerebral média, quando comparados os grupos com RN GIG ou não, em todas as semanas analisadas no presente trabalho. Em relação ao pico de velo- 
cidade sistólica da $\mathrm{ACM}$, não observamos redução significativa de seus valores no grupo com RN GIG, quando comparados ao grupo de RN não GIG nas semanas estudadas.

Tabela 1 - Médias, desvios-padrões (DP) e classificação do índice do líquido amniótico (ILA) como superior ou igual a 18,0 cm ou não, nas idades gestacionais, segundo os grupos GIG (grande para idade gestacional) e não GIG, em gestações com diabete melito pré-gestacional.

\begin{tabular}{|c|c|c|c|c|c|c|c|c|c|}
\hline \multirow{2}{*}{$\begin{array}{c}\text { IG } \\
\text { (semanas) }\end{array}$} & \multirow[t]{2}{*}{$\mathrm{n}$} & \multicolumn{3}{|c|}{$\begin{array}{c}\text { ILA } \\
\text { média e } \pm \mathrm{DP}\end{array}$} & \multicolumn{2}{|c|}{$\begin{array}{c}\text { ILA<18,0 } \\
\text { n (\%) }\end{array}$} & \multicolumn{2}{|c|}{$\begin{array}{c}\text { ILA } \geq 18,0 \\
\text { n (\%) }\end{array}$} & \multirow[b]{2}{*}{$p$} \\
\hline & & Não GIG & GIG & $p$ & Não GIG & GIG & Não GIG & GIG & \\
\hline 28 & 32 & $14,9 \pm 3,9$ & $16,0 \pm 3,0$ & NS & $17(73,9)$ & $6(26,1)$ & $7(77,8)$ & $2(22,2)$ & NS \\
\hline 30 & 38 & $14,9 \pm 3,8$ & $14,6 \pm 3,4$ & NS & $23(71,9)$ & $9(28,1)$ & $5(83,3)$ & $1(16,7)$ & NS \\
\hline 31 & 32 & $13,6 \pm 3,6$ & $14,7 \pm 3,1$ & NS & $20(76,9)$ & $6(23,1)$ & $4(66,7)$ & $2(33,3)$ & NS \\
\hline 32 & 38 & $13,5 \pm 3,6$ & $16,5 \pm 3,5$ & 0,02 & $23(76,7)$ & $7(23,3)$ & $4(50,0)$ & $4(50,0)$ & NS \\
\hline 33 & 35 & $13,1 \pm 4,0$ & $16,7 \pm 5,1$ & 0,03 & $22(78,6)$ & $6(21,4)$ & $4(57,1)$ & $3(42,9)$ & NS \\
\hline 34 & 33 & $13,4 \pm 3,3$ & $17,0 \pm 5,0$ & 0,02 & $19(82,6)$ & $4(17,4)$ & $4(40,0)$ & $6(60,0)$ & $0,03^{*}$ \\
\hline 35 & 38 & $12,8 \pm 3,3$ & $17,9 \pm 3,9$ & 0,000 & $25(80,6)$ & $6(19,4)$ & $2(28,6)$ & $5(71,4)$ & $0,01^{*}$ \\
\hline 36 & 32 & $12,5 \pm 4,0$ & $15,8 \pm 3,3$ & 0,03 & $21(77,8)$ & $6(22,2)$ & $1(20,0)$ & $4(80,0)$ & $0,02^{*}$ \\
\hline 37 & 31 & $12,8 \pm 3,3$ & $17,5 \pm 4,4$ & 0,003 & $20(80,0)$ & $5(20,0)$ & $2(33,3)$ & $4(66,7)$ & $0,04^{*}$ \\
\hline 38 & 21 & $12,4 \pm 5,5$ & $15,7 \pm 4,8$ & NS & $11(68,7)$ & $5(31,3)$ & $2(40,0)$ & $3(60,0)$ & NS \\
\hline
\end{tabular}

NS = não significativo; * comparação RN GIG x RN não GIG

Tabela 2 - Médias e desvios padrões (DP) dos índices de pulsatilidade (IP) das artérias umbilical (AU) e cerebral média (ACM) e da velocidade sistólica máxima da ACM, segundo os grupos GIG (grande para idade gestacional) e não GIG, em gestações com diabete melito pré-gestacional.

\begin{tabular}{|c|c|c|c|c|c|c|c|c|c|c|c|c|}
\hline \multirow{2}{*}{$\begin{array}{c}\text { IG } \\
\text { (semanas) }\end{array}$} & \multicolumn{4}{|c|}{$A U(I P)$} & \multicolumn{4}{|c|}{ ACM (IP) } & \multicolumn{4}{|c|}{ ACM (Vel. Máxima) } \\
\hline & $n$ & Não GIG & GIG & $p$ & $\mathrm{n}$ & Não GIG & GIG & $p$ & $\mathrm{n}$ & Não GIG & GIG & $p$ \\
\hline 28 & 32 & $1,10 \pm 0,15$ & $1,10 \pm 0,19$ & NS & 31 & $2,09 \pm 0,35$ & $1,83 \pm 0,28$ & NS & 31 & $40,6 \pm 8,1$ & $36,5 \pm 8,7$ & NS \\
\hline 30 & 34 & $1,08 \pm 0,14$ & $1,09 \pm 0,20$ & NS & 30 & $2,02 \pm 0,47$ & $2,01 \pm 0,38$ & NS & 30 & $39,6 \pm 11,7$ & $39,4 \pm 16,2$ & NS \\
\hline 31 & 28 & $1,02 \pm 0,19$ & $1,00 \pm 0,13$ & NS & 26 & $1,90 \pm 0,37$ & $2,00 \pm 0,37$ & NS & 26 & $38,5 \pm 12,6$ & $42,5 \pm 11,2$ & NS \\
\hline 32 & 42 & $1,04 \pm 0,15$ & $1,06 \pm 0,17$ & NS & 40 & $1,90 \pm 0,32$ & $2,02 \pm 0,45$ & NS & 40 & $48,2 \pm 16,8$ & $45,3 \pm 14,9$ & NS \\
\hline 33 & 34 & $1,02 \pm 0,16$ & $1,19 \pm 0,33$ & NS & 31 & $1,92 \pm 0,45$ & $1,97 \pm 0,50$ & NS & 31 & $46,4 \pm 13,8$ & $41,7 \pm 11,3$ & NS \\
\hline 34 & 30 & $0,98 \pm 0,19$ & $1,04 \pm 0,17$ & NS & 30 & $1,87 \pm 0,38$ & $1,95 \pm 0,68$ & NS & 30 & $45,4 \pm 20,1$ & $44,8 \pm 15,1$ & NS \\
\hline 35 & 37 & $0,95 \pm 0,13$ & $0,93 \pm 0,16$ & NS & 36 & $1,73 \pm 0,26$ & $1,72 \pm 0,48$ & NS & 36 & $48,6 \pm 16,5$ & $46,6 \pm 19,6$ & NS \\
\hline 36 & 31 & $1,05 \pm 0,22$ & $1,01 \pm 0,29$ & NS & 30 & $1,78 \pm 0,35$ & $1,58 \pm 0,36$ & NS & 30 & $55,4 \pm 11,2$ & $54,6 \pm 13,4$ & NS \\
\hline 37 & 28 & $0,92 \pm 0,16$ & $1,01 \pm 0,25$ & NS & 26 & $1,58 \pm 0,40$ & $1,55 \pm 0,49$ & NS & 26 & $49,9 \pm 14,9$ & $48,0 \pm 16,4$ & NS \\
\hline 38 & 21 & $0,90 \pm 0,20$ & $1,00 \pm 0,23$ & NS & 22 & $1,37 \pm 0,28$ & $1,56 \pm 0,53$ & NS & 22 & $49,8 \pm 22,8$ & $47,1 \pm 13,6$ & NS \\
\hline
\end{tabular}

NS = não significativo.

Os resultados perinatais na população analisada não apresentaram diferenças significativas segundo a adequação do peso do RN. Quatro $(8,7 \%)$ $\mathrm{RN}$ apresentaram indice de Apgar de $1^{\circ}$ minuto inferior a 7 , sendo que um era GIG e três não. Todos os RN apresentaram indices de Apgar de $5^{\circ}$ e $10^{\circ}$ minuto com valores superiores a 7 . Três RN $(6,5 \%)$ necessitaram de internação em unidade de terapia intensiva neonatal, sendo que um destes era GIG. Não houve diferenças na distribuição segundo o sexo do RN, sendo que 55\% (11 casos) eram do sexo masculino no grupo AIG ou PIG e $47 \%$ (7 casos) no grupo GIG.

\section{Discussão}

A prevenção da morte fetal em gestações de mães diabéticas têm sido realizada, com eficiência, ao associar-se o controle clínico materno rigoroso aos métodos propedêuticos de avaliação 
fetal. Aparentemente, o adequado controle metabólico, na ausência de vasculopatias ou outras intercorrências clínicas e/ou obstétricas, raramente resulta em sofrimento fetal na gestação com diabete melito ${ }^{11}$. Kjos et al. ${ }^{17}$, analisando 1501 gestações em pacientes com diabete melito, referem que 2,2\% dos testes realizados apresentaram anormalidades que resultaram na resolução da gestação. Lagrew et al. ${ }^{18}$, analisando todas as gestantes diabéticas do seu serviço no período entre 1981 e 1991, referem que em $11,6 \%$ das pacientes a resolução da gravidez foi indicada por apresentarem resultados anormais na avaliação fetal, e sugerem que se inicie a vigilância do bem-estar fetal na $32^{\mathrm{a}}$ semana. Na nossa casuística, apenas em dois casos de gestantes com diabete melito pré-gestacional $(4,6 \%)$ o PBF apresentou resultado de 6 , indicando resolução da gestação.

O controle clínico materno em gestações com diabete melito tem proporcionado, no nosso serviço, baixa incidência de alterações nos testes de vitalidade que caracterizem o sofrimento fetal. Porém, observamos elevada incidência de RN GIG, o que reflete a necessidade de novos métodos que auxiliem no ajuste rigoroso das condições metabólicas materno-fetais e que proporcionem melhores condições ao nascimento. A morbidade neonatal nas primeiras horas de vida do RN de mãe diabética inclui não somente episódios de hipoglicemia, mas também outras anormalidades tais como hipocalcemia, hiperbilirrubinemia, cardiomegalia e taquipnéia transitória ${ }^{5}$. Além disto, sabe-se que aumentam os riscos de complicações durante o trabalho de parto quando o feto apresenta macrossomia ${ }^{11}$.

O seguimento longitudinal realizado neste estudo demonstrou que o índice do líquido amniótico representa um papel importante como marcador do descontrole metabólico fetal. O polidrâmnio é relatado como complicação do diabete melito na gravidez, e a sua fisiopatologia envolve a hiperglicemia fetal provocando a diurese osmótica e conseqüente acúmulo de líquido na cavidade amniótica ${ }^{10}$. Dashe et al. ${ }^{19}$, em 2000, demonstraram correlação positiva entre os valores do ILA e a concentração de glicose no líquido amniótico em gestantes diabéticas, reforçando portanto esta hipótese. O nosso estudo demonstra, ao realizar o seguimento longitudinal de gestantes diabéticas pré-gestacionais, que valores do ILA superiores a $18,0 \mathrm{~cm}$, principalmente entre a $34^{\mathrm{a}}$ e a $37^{\mathrm{a}}$ semana da gestação, refletem controle inadequado do metabolismo fetal, que irá se refletir no nascimento de RN GIG.

A definição utilizada para o diagnóstico de polidrâmnio pelo ILA refere-se a valores superiores a $25,0 \mathrm{~cm}^{20}$. Porém, observamos que este ní- vel de corte apenas discrimina os casos mais graves, geralmente associados a anomalias fetais. Graus intermediários de aumento do volume de líquido amniótico podem ser caracterizados a partir da classificação proposta por Rutherford et al. ${ }^{14}$ para o índice. Estes autores, inicialmente, propunham o limite de $18,0 \mathrm{~cm}$ para o diagnóstico de polidrâmnio. Kjos et al. ${ }^{17}$ não observaram associação entre os valores do ILA e do diâmetro do maior bolsão de líquido amniótico, com a ocorrência de sofrimento fetal. Entretanto, este estudo não correlaciona a avaliação do volume de líquido amniótico com o descontrole metabólico fetal.

Evidentemente, o período em que é observado o aumento do ILA também irá influenciar o padrão do crescimento fetal. Em alguns casos, observamos que valores de ILA elevados, que foram compensados com o progredir da gestação, associaram-se a recém-nascidos de peso adequado ao nascimento. Somente o acompanhamento prospectivo de maior número de pacientes permitirá definir em quais pacientes as faixas dos valores do ILA e os padrões de elevação dos mesmos irão orientar os ajustes na insulinoterapia.

Um dos aspectos interessantes na utilização do ILA como um parâmetro de checagem do controle glicêmico materno reside no fato de que os mecanismos de troca do líquido amniótico são extremamente dinâmicos, refletindo rapidamente as alterações metabólicas que ocorrem na gestação ${ }^{10}$. Outras medidas indiretas do crescimento excessivo fetal, tais como o aumento da circunferência abdominal, ou de outros marcadores indiretos de depósito aumentado da gordura no feto, não apresentam características tão dinâmicas como o acúmulo do líquido amniótico não permitindo que medidas sejam adotadas em tempo hábil.

A avaliação da dopplervelocimetria da artéria umbilical em gestantes diabéticas não têm demonstrado associação da doença com anormalidades da circulação placentária ou fetal ${ }^{9,21}$. Porém, quando ocorre associação com outras anormalidades tais como a hipertensão ou a vasculopatia, esta técnica tem papel fundamental na avaliação da função placentária ${ }^{22,23}$. Bracero et al. $^{22}$ relatam associação entre indices elevados na relação sístole/diástole da artéria umbilical em gestantes diabéticas com aumento nas taxas de natimortalidade e de morbidade neonatal. Landon et al. ${ }^{23}$ concluem que pacientes diabéticas com vasculopatia (classe F/R ou com hipertensão arterial crônica) representam um grupo de alto risco para restrição do crescimento fetal. No presente estudo, a análise longitudinal da dopplervelocimetria da artéria umbilical não apresentou associação significativa com a ocorrência de RN GIG. 
Entretanto, a placenta da mãe diabética apresenta alterações morfológicas que podem influenciar a resistência do leito placentário, bem como o seu padrão de perfusão ${ }^{24}$.

A dopplervelocimetria da artéria cerebral média também não apresentou alterações significativas nesta casuística. Do mesmo modo, Salvesen et al. ${ }^{9}$ não puderam demonstrar alterações no fluxo da aorta fetal e da artéria cerebral média, a despeito do aumento dos niveis de hematócrito constatado no sangue fetal colhido por cordocentese. Os autores sugerem que seriam necessárias graves alterações na viscosidade sangüinea para que fossem detectadas anormalidades. Deve-se considerar que a metodologia utilizada por estes autores (velocidade média) difere da apresentada neste estudo, no qual foi avaliado o pico de velocidade máxima. Degani et al. ${ }^{25}$, em 1991, avaliaram o fluxo na artéria carótida interna após a ingestão de $100 \mathrm{~g}$ de glicose, e observaram associação entre aumento do indice de pulsatilidade e aumento da glicemia. Os autores sugerem a existência de um mecanismo de autoregulação da resistência vascular cerebral que é ativado pelos níveis glicêmicos. Os dados do nosso trabalho não permitiram correlacionar alterações na ACM com a condição metabólica fetal, porém, estudos mais detalhados serão conduzidos para melhor compreensão destes mecanismos.

Este estudo permite concluir que alterações do volume de líquido amniótico caracterizadas pelo aumento dos valores do ILA, principalmente aqueles superiores a $18,0 \mathrm{~cm}$, estão relacionadas ao nascimento de RN GIG e, conseqüentemente, ao descontrole metabólico fetal. Assim, ajustes na terapêutica materna devem ser recomendados e reavaliados para que se obtenha o melhor resultado possivel para o binômio mãefeto, de acordo com os valores do ILA calculados durante a gestação. Não foi possível, com a presente casuística, correlacionar o descontrole metabólico fetal com os valores da velocidade sistólica máxima avaliada na dopplervelocimetria da artéria cerebral média fetal.

\section{ABSTRACT}

Purpose: to study fetal surveillance examinations in pregnancies complicated by pregestational diabetes mellitus, and to correlate them with large for gestational age (LGA) newborns.

Methods: Between March 1999 and June 2001, 46 singleton pregnancies with pregestational diabetes mellitus without fetal anomalies were followed prospectively. From the $28^{\text {th }}$ gestational week on, the following examinations were performed weekly: fetal biophysical profile, amniotic fluid index (AFI), and dopplervelocimetry of umbilical and middle cerebral arteries. The newborns with birthweight above the $90^{\text {th }}$ percentile according to local standard values were characterized as LGA infants. Fisher's exact test and Student's $\mathrm{t}$ test were used for statistical analysis.

Results: The mean gestational age at delivery was 37.6 weeks and 15 (32.6\%) newborns were LGA. LGA fetuses showed significant increase in the AFI mean performed in the $32^{\text {nd }}$ (16.5 cm, $p=0.02), 33^{\text {rd }}(16.7 \mathrm{~cm}, p=0.03), 34^{\text {th }}(17.0 \mathrm{~cm}$, $p=0.02), 35^{\text {th }}(17.9 \mathrm{~cm}, p=0.000), 36^{\text {th }}(15.8 \mathrm{~cm}, p=0.03)$ and $37^{\text {th }}(17.5 \mathrm{~cm}, p=0.003)$ weeks. Non-LGA fetuses presented the following mean AFI values: $13.5 \mathrm{~cm}$ (32 $2^{\text {nd }}$ week), $13.1 \mathrm{~cm}$

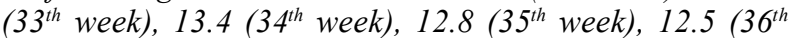
week) and $12.8 \mathrm{~cm}\left(37^{\text {th }}\right.$ week). AFI values equal to or above $18.0 \mathrm{~cm}$ were associated with the occurrence of LGA infants, when detected at the following gestational ages: $34^{\text {th }}(60 \%$, $p=0.03), 35^{\text {th }}(71.4 \%, p=0.01), 36^{\text {th }}(80 \%, p=0.02)$ and $37^{\text {th }}$ (66.7\%, $p=0.04)$ week. Non-LGA infants presented the following proportion of AFI values equal to or above 18.0 $\mathrm{cm}: 40.0 \%\left(34^{\text {th }}\right.$ week $), 28.6 \%\left(35^{\text {th }}\right.$ week $), 20.0 \%$ (36 $6^{\text {th }}$ week), and $33.3 \%$ (37 $7^{\text {th }}$ week).

Conclusions: abnormal increase in AFI, mainly with values equal to or above $18.0 \mathrm{~cm}$, is related to LGA infants at delivery. The maternal treatment should be adjusted to achieve the best result for maternal-fetal control, according to the AFI values during pregnancy.

KEY WORDS: Diabetes mellitus. Amniotic fluid index. Fetal biophysical profile. Dopplervelocimetry. Umbilical artery. Middle cerebral artery.

\section{Referências Bibliográficas}

1. Felig P, Coustan D. Diabetes mellitus. In: Ferris B, editor. Complicações Clínicas na Gravidez. $2^{\mathrm{a}}$ ed. São Paulo: Roca; 1983. p.35-59.

2. White P. Pregnancy complicating diabetes. Am J Med $1949 ; 7: 609-16$.

3. Harman CR, Menticoglou SM. Fetal surveillance in diabetic pregnancy. Curr Opin Obstet Gynecol 1997; 9:83-90.

4. Jovanovic-Peterson L, Peterson CM. The art and science of maintenance of normoglycemia in pregnancies complicated by insulin-dependent diabetes mellitus. Endocr Pract 1996; 2:130-43.

5. Jovanovic L, Peterson CM, Saxena BB, Dawood MY, Saudek CD. Feasibility of maintaining normal glucose profiles in insulin-dependent pregnant diabetic women. Am J Med 1980; 68:105-12.

6. Zugaib M, Kanas M. Crescimento fetal. In: Zugaib M, Kanas M, editores. Fisiologia Fetal Aplicada. $1^{\mathrm{a}}$ ed. São Paulo: Roca; 1986. p.149-68. 
7. Mintz DH, Skyler JS, Chez RA. Diabetes mellitus and pregnancy. Diabetes Care 1978; 1:49-63.

8. Philipps AF, Dublin JW, Matty PJ, Raye JR. Arterial hypoxemia and hyperinsulinemia in the chronically hyperglycemic fetal lamb. Pediatr Res 1982; 16:653-8.

9. Salvesen DR, Higueras MT, Mansur CA, Freeman J, Brudenell JM, Nicolaides KH. Placental and fetal Doppler velocimetry in pregnancies complicated by maternal diabetes mellitus. Am J Obstet Gynecol 1993; 168:645-52.

10.Cardwell MS. Polyhydramnios: a review. Obstet Gynecol Surv 1987; 42:612-7.

11.Landon MB, Gabbe SG. Fetal surveillance in the pregnancy complicated by diabetes mellitus. Clin Obstet Gynecol 1991; 34:535-43.

12.Yamamoto RM, Francisco RPV, Miyadahira S, Banduki Neto JD, Zugaib M. Avaliação da vitalidade fetal em gestantes diabéticas: análise dos resultados neonatais. Rev Bras Ginecol Obstet 2000; 22:557-66.

13. Phelan JP, Smith CV, Broussard P, Small M. Amniotic fluid volume assessment with the fourquadrant technique at 36-42 weeks' gestation. J Reprod Med 1987; 32:540-2.

14.Rutherford SE, Phelan JP, Smith CV, Jacobs N. The four-quadrant assessment of amniotic fluid volume: an adjunct to antepartum fetal heart rate testing. Obstet Gynecol 1987; 70:353-6.

15. Mari G, Deter RL. Middle cerebral artery flow velocity waveforms in normal and small-for-gestationalage fetuses. Am J Obstet Gynecol 1992; 166:1262-70.

16.Ramos JLA. Avaliação do crescimento intra-uterino por medidas antropométricas do recém-nascido [tese]. São Paulo: Universidade de São Paulo; 1983.
17.Kjos SL, Leung A, Henry OA, Victor MR, Paul RH, Medearis AL. Antepartum surveillance in diabetic pregnancies: predictors of fetal distress in labor. Am J Obstet Gynecol 1995; 173:1532-9.

18.Lagrew DC, Pircon RA, Towers CV, Dorchester W, Freeman RK. Antepartum fetal surveillance in patients with diabetes: when to start? Am J Obstet Gynecol 1993; 168:1820-6.

19.Dashe JS, Nathan L, McIntire DD, Leveno KJ. Correlation between amniotic fluid glucose concentration and amniotic fluid volume in pregnancy complicated by diabetes. Am J Obstet Gynecol 2000; 182:901-4.

20.Carlson DE, Platt LD, Medearis AL, Horenstein J. Quantifiable polyhydramnios: diagnosis and management. Obstet Gynecol 1990; 75:989-93.

21.Bem-Ami M, Battino S, Geslevich Y, Shalev E. A random single Doppler study of the umbilical artery in the evaluation of pregnancies complicated by diabetes. Am J Perinatol 1995; 12:437-8.

22.Bracero L, Schulman H, Fleischer A, Farmakides G, Rochelson B. Umbilical artery velocimetry in diabetes and pregnancy. Obstet Gynecol 1986; 68:654-8.

23.Landon MB, Gabbe SG, Bruner JP, Ludmir J. Doppler umbilical artery velocimetry in pregnancy complicated by insulin-dependent diabetes mellitus. Obstet Gynecol 1989; 73:961-5.

24.Bjork O, Persson B. Placental changes in relation to the degree of metabolic control in diabetes mellitus. Placenta 1982; 3:367-78.

25.Degani S, Paltieli Y, Gonen R, Sharf M. Fetal internal carotid artery pulsed Doppler flow velocity waveforms and maternal plasma glucose levels. Obstet Gynecol 1991; 77:379-81.

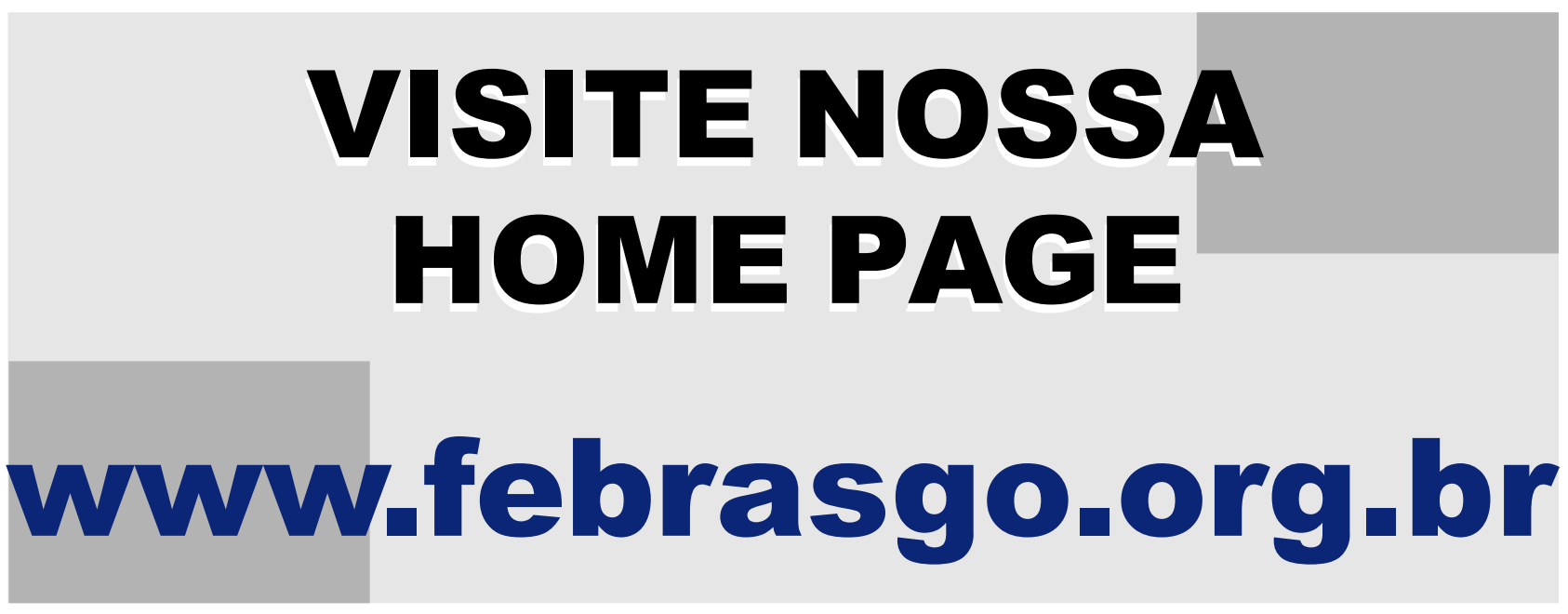

